\title{
Successful Treatment of Aortic Rupture with Endovascular Stent Grafting in a Patient with Mantle Cell Lymphoma
}

\author{
A.T. Sumbul ${ }^{a} \quad$ F. Kose ${ }^{a} \quad$ L. Oğuzkurt ${ }^{b} \quad$ C. Karadeniz ${ }^{a}$ \\ N.E. Kocer ${ }^{c}$ O. Ozyilkan ${ }^{a}$
}

Departments of ${ }^{\mathrm{a}}$ Medical Oncology, ${ }^{\mathrm{b}}$ Interventional Radiology and ${ }^{\mathrm{c}}$ Pathology, Faculty of Medicine, Baskent University, Ankara, Turkey

\section{Key Words}

Systemic chemotherapy · Abdominal aortic rupture - Lymphoma

Endovascular stent-graft repair

\begin{abstract}
Purpose: To present a case of spontaneous aortic rupture in the course of mantle cell lymphoma and successful management with endovascular repair.
\end{abstract}

Case Report: A 69-year-old woman presented with a cervical mass. The patient was found to have stage IIIA and Mantle Cell Lymphoma International Prognostic Index (MIPI) 4. She was placed in an intermediate-risk group. The patient received an initial cycle of systemic chemotherapy consisting of rituximab, anthracycline, vincristine and methyl prednisolone. During follow-up, she developed abdominal aortic rupture secondary to intramural hematoma which was successfully managed with endovascular exclusion.

Conclusion: Hemodynamic changes can be seen during the course of lymphoma subsequent to systemic chemotherapy. These changes might be related to the spontaneous rupture of the main vessels. Endovascular repair may be a more appropriate treatment option than open surgery, especially in a patient with multiple comorbidities.

\section{Introduction}

Potential causes of aortic rupture are aneurysm, trauma and plaque rupture. Aneurysmal rupture during the course of systemic chemotherapy is an unusual and serious complication which is difficult to manage, especially in a patient whose health is compromised because of cancer and comorbid conditions. Specific therapeutic modalities have to be coordinated. One of the novel developments in interventional 
radiology practice is endoluminal aortic stent grafting in the management of aortic aneurysm including aneurysmal rupture. The first encouraging experiences were published by Parodi et al. in 1991 [1]. Therein, the authors report a case with abdominal aortic rupture during the course of systemic chemotherapy and successful treatment with endovascular stent grafting in an urgent care setting.

\section{Case Report}

A 69-year-old woman presented with a cervical mass. She had been evaluated at an independent medical center and a cervical excisional biopsy had been performed. The patient was referred to our Medical Oncology Department with a pathology report. The patient had a history of mild hypertension with no need for medication. She had no history of weight loss, night fever or excessive sweating. Her physical examination was unremarkable except for cervical lymphadenopathy and obesity. Histopathological discussion of the biopsy revealed a diagnosis of mantle cell lymphoma. The patient was staged with PET-CT, endoscopic examination of the gastrointestinal system and bone marrow aspiration/biopsy. PET-CT showed multiple hypermetabolic foci at the cervical, mediastinal and retroperitoneal regions. The patient was staged as IIIA. MIPI (Mantle Cell Lymphoma International Prognostic Index) of the patient was 4, so she was placed in the intermediate-risk group. The treatment plan consisted of 6 cycles of R-CHOP regimen. Two days after the first cycle of systemic chemotherapy, syncope occurred, blood pressure was $70 / 50 \mathrm{~mm} \mathrm{Hg}$, and her hematocrit level was $27 \%$; the other parts of the complete blood count were normal, as were the results of other laboratory tests, including serum electrolytes and tests for renal and liver function. Cardiological and surgical evaluations were normal.

The patient was transferred to the Intensive Care Unit. Over the next 2 days, her hematocrit values progressively decreased and 6 units of packed red blood cells were transfused. Vital signs remained normal during this period. Thoracic and abdominal CT scans were performed, revealing a huge intramural hematoma and a retroperitoneal hematoma (fig. 1). The retroperitoneal hematoma was secondary to the intramural hematoma. Her aorta did not seem aneurysmatic. Cardiovascular Surgery and the Interventional Radiology Unit consulted on the patient. Due to comorbid conditions, an open surgical procedure was considered to have high preoperative and postoperative mortality; thus the patient was transferred to the Interventional Radiology Unit. A percutaneous approach was used from the right and left common femoral arteries. Diagnostic angiography revealed that the distal aorta had minimal aneurysmatic dilatation which was very mild at $30 \mathrm{~mm}$ in diameter (fig. $2 \mathrm{a}$ ). A 28-mm stent graft (Medtronic, USA) was placed into the aorta, and then the contralateral leg was placed. A check angiogram showed excellent positioning of the stent graft with no leak (fig. 2b). The patient's hemoglobin level stabilized immediately after the stent-graft placement.

\section{Discussion}

The most frequent etiology of aortic rupture is aneurysm. Risk of rupture of an aortic aneurysm increases with increased diameter of the aorta. Currently, a diameter more than $55 \mathrm{~mm}$ is the indication for treatment with surgical or endovascular means. There are other potential causes such as trauma and plaque rupture. Nontraumatic, noninfectious rupture of the nonaneurysmal aorta is a very rare condition and the most frequent cause is penetrating atherosclerotic ulcer [2]. Abdominal aortic aneurysms (AAA) are common and generally asymptomatic unless rupture occurs. A 3-4-cm AAA has a 1-2\% risk of rupture over 5 years [3]. Aneurysms in oncologic cases are mostly seen with colorectal carcinomas and usually located in the abdominal aorta. Aneurysmal and nonaneurysmal rupture of the aorta in oncologic cases is a very rare condition and mostly seen in patients who have tumors with periaortic localization and concomitant aneurysmal dilatation [4]. For primary aortic tumors, rupture has been 
described only in a single case of leiomyosarcoma of the thoracic aorta [5]. Ehata et al. [6] reported a case of ruptured AAA repair in which a pheochromocytoma was diagnosed postoperatively by CT scan. Thompson et al. [7] also reported a case in which a pheochromocytoma was found using metaiodobenzylguanidine imaging after a repaired AAA began leaking. Moreover, 4 cases of dissecting thoracic aneurysms associated with pheochromocytoma have been described [6]. Spontaneous rupture of the nonaneurysmal aorta has been reported with tumor infiltration in neurofibromatosis [8]. All reported cases of primary malignant lymphoma invading the aorta were associated with symptomatic or ruptured aortic aneurysms. Aortoiliac fistula was found in 1 case and endovascular repair was successfully performed [9].

Penetrating ulcerated plaque and hemodynamic changes such as hypertension are the most common causes of aneurysmal rupture of the aorta. Chemotherapy is a stress factor for hemodynamic parameters, but the patient presented here received her systemic chemotherapy in the oncology ward due to the risk of probable tumor lysis syndrome. During in-ward follow-up, no abnormalities were detected by means of physical examination, vital signs and laboratory results. An abdominal CT scan was performed as part of the initial staging, and radiological evaluation showed no abnormality in the abdominal aorta. A repeat CT scan at the time of diagnosis of rupture did not show an aneurysmal aorta but a big intramural hematoma. Diagnostic angiography at the beginning of endovascular intervention showed a very mild dilatation of the distal abdominal aorta.

At this point, the patient could be managed by either open surgery or endovascular stent grafting. Endovascular stent grafting has been shown to reduce procedure-related and early morbidity and mortality in a large prospective randomized trial [10]. Open surgery had the disadvantages of bleeding and infections due to cytopenia and may be hazardous because of poor conditional status and comorbid conditions of the patient. So we decided on endovascular stent grafting.

Endovascular repair provides a valuable alternative in the acute setting of aneurysmal rupture and should be an established treatment option, especially in patients with several comorbid conditions. 


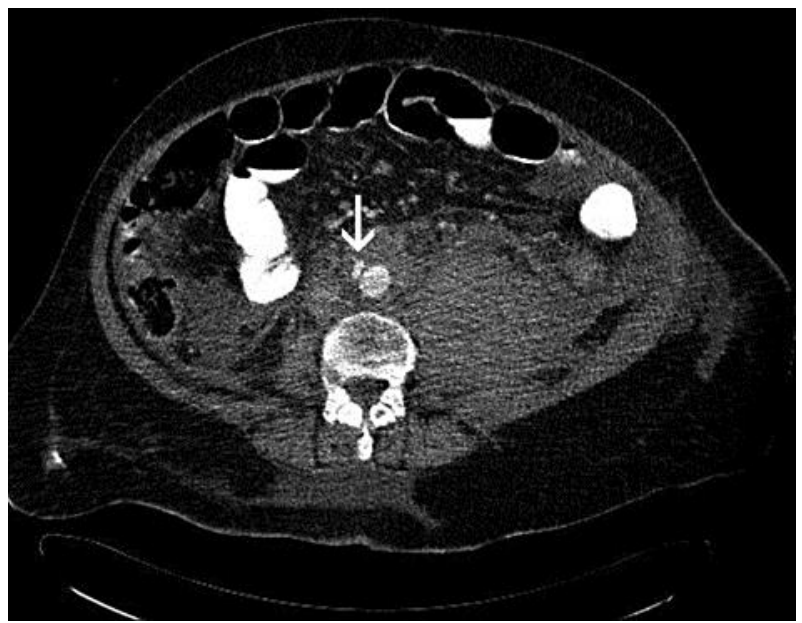

Fig. 1. Contrast-enhanced CT image reveals significant leak from the aorta (arrow). There is a crescent-shaped hematoma in the aortic wall causing the rupture, and a more significant hematoma in the retroperitoneum.
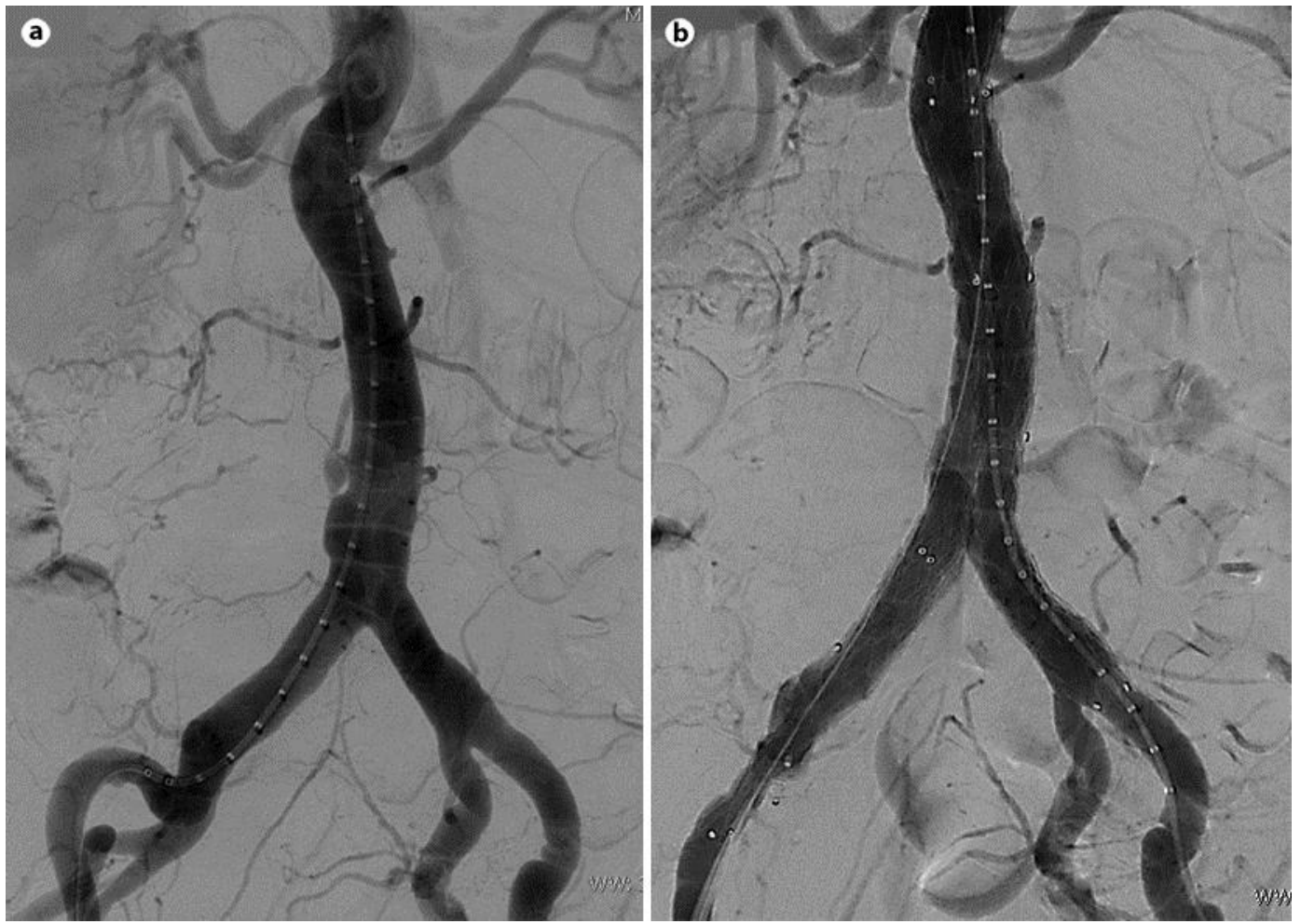

Fig. 2. a Aortogram before stent-graft placement reveals than the aorta is otherwise normal in size. There is a mild widening of the aorta on its left distal side. A leak is not visible on the aortogram. b Aortogram after stent-graft placement. The stent graft seals the whole infrarenal aorta excluding the rupture point. 


\section{References}

1 Parodi JC, Palmaz JC, Barone HD: Transfemoral intraluminal graft implantation for abdominal aortic aneurysms. Ann Vasc Surg 1991;5:491-499.

-2 Batt M, Haudebourg P, Planchard PF, Ferrari E, Hassen Khodja R, Bouilanne PJ: Penetrating atherosclerotic ulcers of the infrarenal aorta: life-threatening lesions. Eur J Vasc Endovasc Surg 2005;29:35-42.

-3 Brandt M, Walluscheck KP, Jahnke T, Graw K, Cremer J, Müller-Hülsbeck S: Endovascular repair of ruptured abdominal aortic aneurysm: feasibility and impact on early outcome. J Vasc Interv Radiol 2005;16:1309-1312.

-4 Ruckert RI, Pirlich M, Rogalla P, Ismail M, Muller JM: Successful endovascular treatment of infrarenal aortic rupture after chemotherapy of a mesenchymal periaortic tumor. Eur J Vasc Endovasc Surg 2007;33:302-305.

5 Bush RL, Lumsden AB, Dodson TF, et al: Mid-term results after endovascular repair of the abdominal aortic aneurysm. J Vasc Surg 2001;33:S70-S76.

-6 Ehata T, Karasawa F, Watanabe K, Satoh T: Unsuspected pheochromocytoma with abdominal aortic aneurysm - a case report. Acta Anaesthesiol Sin 1999;37:27-28.

7 Thompson JF, Clifford PC, McEwan JA, Chant AD: Pheochromocytoma and abdominal aneurysm: confirmation of diagnosis after aortic surgery using MIBG imaging. Eur J Vasc Surg 1989;5:457-459.

8 Hines GL, Lefkowitz L, Mohtashemi M: Infrarenal aortic rupture secondary to neurofibromatosis. Ann Vasc Surg 2002;16:784-786.

-9 Williamson AE, Annunziata G, Cone LA, Smith J: Endovascular repair of a ruptured abdominal aortic and iliac aneurysm with an acute iliocaval fistula secondary to lymphoma. Ann Vasc Surg 2002;16:145-149.

10 Greenhalgh RM, Brown LC, Kwong GP, Powell JT, Thompson SG; EVAR trial participants: Comparison of endovascular aneurysm repair with open repair in patients with abdominal aortic aneurysm (EVAR trial 1), 30-day operative mortality results: randomised controlled trial. Lancet 2004;364:843-848. 